\section{Cerebral arterial air embolism with anterior spinal cord syndrome after CT-guided hook-wire localization of Lung mass and pulmonary nodule}

\author{
Noor Sameh Darwich ${ }^{1 *}$, Umran Ugur ${ }^{2}$, Mark P Anstadt ${ }^{3}$ and \\ Michael J Pedoto ${ }^{4}$
}

\author{
${ }^{1}$ Miami Valley Hospital, Dayton, OH, USA \\ ${ }^{2}$ Department of Neurology and Neuroscience, Wright State University, Miami Valley Hospital, \\ Dayton, $\mathrm{OH}, \mathrm{USA}$ \\ ${ }^{3}$ Department of Thoracic Surgery, Wright State University, Miami Valley Hospital, Dayton, OH, USA \\ ${ }^{4}$ Department of Physical Medicine and Rehabilitation, Miami Valley Hospital, Dayton, OH, USA
}

\section{Abstract}

Systemic arterial air embolism (SAAE) is a rare but serious complication of CT-guided hook wire localization of pulmonary nodule usually with catastrophic and poor outcome. Hook wire needle localization is done pre-operatively by placing wire around or into the pulmonary nodule to provide the thoracic surgeon accurate location guidance of the target nodule for Video-Assisted Thoracoscopic Surgery (VATS) wedge resection with safety margins. Physicians should be aware of this possible complication during the procedure in order to rescue the patient promptly as it requires rapid diagnosis and management. We describe a 55-year-old male who underwent a CT-guided hook wire needle localization of left upper lobe lung cancer and left lower lobe pulmonary nodule prior to planned VATS wedge resection who developed altered mental status and bilateral lower extremities paralysis after wire placement was completed. His CT head demonstrated small air embolism in the left occipital area, confirming the diagnosis of cerebral air embolism and follow up CT and MRI of the head revealed multiple areas of brain infarction. In addition, he was diagnosed with anterior spinal cord syndrome (ACS), most likely due to anterior spinal artery ischemia from micro air embolism on the basis of clinical findings but with negative ischemic changes on MRI of the spinal cord. His mental status recovered but he remained paraplegic and transferred to inpatient rehabilitation service.
More Information

*Address for Correspondence: Noor Sameh Darwich, Miami Valley Hospital, One Wyoming Street, Dayton, OH 45409, USA, Email: nsdarwich@gmail.com

Submitted: March 02, 2021

Approved: March 18, 2021

Published: March 19, 2021

How to cite this article: Darwich NS, Ugur U, Anstadt MP, Pedoto MJ. Cerebral arterial air embolism with anterior spinal cord syndrome after CT-guided hook-wire localization of Lung mass and pulmonary nodule. J Neurosci Neurol Disord. 2021; 5: 006-015.

DOI: 10.29328/journal.jnnd.1001044

Copyright: @ 2021 Darwich NS, et al. This is an open access article distributed under the Creative Commons Attribution License, which permits unrestricted use, distribution, and reproduction in any medium, provided the original work is properly cited.

Keywords: Cerebral arterial air embolism; Hookwire localization; Anterior spinal cord syndrome (ACS); Systemic arterial air embolism (SAAE)

Check for updates

OPEN ACCESS

\section{Introduction}

Systemic arterial air embolism (SAAE) after interventional pulmonary procedures, including CT-guided lung biopsy and Hook-wire needle localization of pulmonary nodule, is an extremely rare complication. However, the actual incidence is likely to be higher because a significant number of patients remain asymptomatic or undiagnosed after its occurrence and delayed presentation is not uncommon [1,2]. The brain, heart and spinal cord are the organs mostly affected by ischemia induced by SAAE. Patients with cerebral air embolism may present with altered mental status, seizures, dysarthria, visual field defect, and limb paralysis. Likewise, anterior spinal cord syndrome (ACS) due to SAAE is a rare occurrence with diagnostic challenges [3]. ACS, also known as Anterior spinal artery syndrome, presents with sudden onset of bilateral loss in motor functions of the lower extremities. Moreover, it is also featured by loss of pain and temperature sensation but sparing of the proprioceptive and vibratory sensations occurring below the level of the ischemic lesion in the spinal cord with autonomic dysfunction primarily manifest as bowel and bladder incontinence as well as sexual dysfunction and orthostatic hypotension [4]. Although the actual prevalence of SAAE remains unknown, its prognosis is very poor [5]. There is uncertainty regarding the underlying mechanism of systemic arterial air embolism (SAAE) after hook-wire needle localization of pulmonary nodule. Preventive measures and early recognition of SAAE are important to improve patient outcomes and reduce mortality. Initial treatment is usually limited to supportive care which involves administration of $100 \% \mathrm{FIO}_{2}$ by face mask while hyperbaric oxygen therapy (HBOT) is considered the optimal first line treatment for 
SAAE as it helps to mitigate ischemic injuries to the affected organs [6]. The optimal position for patient with SAAE remains controversial, however the current recommendation is to place the patient in a supine position [7]. High level of awareness among physicians performing these invasive procedures for the possibility of SAAE as a potential complication is crucial [8].

\section{Case report}

A 55-year-old male with a recent history of right VATS lower lobectomy for stage II (T2N1M0) poorly differentiated adenocarcinoma of the lung presented with a $3.1 \mathrm{x}$ $2.5 \times 2.7 \mathrm{~cm}$ left upper lobe (LUL) lung mass. The mass was PET avid and was deemed to be second primary lung cancer after a CT- guided lung biopsy demonstrating fairly well-differentiated adenocarcinoma of the lung with lipidic pattern. He was also diagnosed with an indeterminate $8 \mathrm{~mm}$ left lower lobe lung nodule. CT-guided hook wire needle localization of both lung lesions before left VATS wedge resection of the LUL lung cancer and the left lower lobe nodule was planned. At the start of the procedure, the patient was alert and oriented with normal vitals and physical exam with no focal deficits noted and his routine laboratory tests were within normal limits. He first underwent epidural catheter placement for post VATS pain management with successful and safe insertion of the catheter at T7-T8 level with the use of only local lidocaine. No testing dose of Fentanyl was used after placement of the catheter and there were no changes in the neurological status after the procedure following epidural catheter placement.

The patient was initially placed supine on the CT table and computed tomography images were obtained which showed no pneumothorax but only residual left sided chest wall subcutaneous emphysema and small pneumomediastinum resulted from recent mediastinoscopy and CT- guided left upper lobe lung biopsy done three weeks earlier, which appeared much improved compared to previous study, as well as an epidural catheter noted with few intraspinal gas bubbles adjacent to the catheter insertion site. A Remington hook wire was deployed in the left upper lung mass through a 20-gauge needle (Figure 1) without difficulty but with small left pneumothorax.

The patient was then placed in a prone position, and a 20-gauge Remington needle was advanced to the left lower lobe nodule which led to some nodular hemorrhage as noted from the images after needle placement while the hook wire was further advanced and placed without difficulty. At this stage, it was noted that the patient was not moving his right side, and then he became unresponsive and hypertensive. He was immediately placed back in the supine position and supplemented with $100 \% \mathrm{FIO}_{2}$ by face mask. Upon the arrival of the stroke alert team, the patient was answering all questions and following all commands albeit slowly. He had mild left arm drift, right visual field deficit, and paraplegia. He had a score of 12 on the NIH stroke scale (Table 1).

He was resuscitated and was placed in a Trendelenburg position as there was a concern for potential cerebral air embolus. CT head without contrast was done 26 minutes after the end of the procedure showed a small hypodense area in the left occipital cortex that correlate with an air embolus but no hemorrhage, mass effect or midline shift was noted (Figure 2). CT thoracic spine showed subcutaneous and epidural gas at

\begin{tabular}{|c|c|}
\hline 1a Level of Consciousness & $\begin{array}{l}1 \text { - Not alert, but arousable by minor stimulation } \\
\text { to obey, answer, or respond }\end{array}$ \\
\hline 1b LOC Questions & 0 - Answers both questions correctly \\
\hline 1c LOC Commands & 0 - Performs both tasks correctly \\
\hline 2 Best Gaze & $\begin{array}{c}1 \text { - Partial gaze palsy, the gaze is abnormal in } \\
\text { one or both eyes but forced deviation and total } \\
\text { gaze paresis is not present }\end{array}$ \\
\hline 3 Visual & 1 - Partial hemianopsia \\
\hline 4 Facial Palsy & 0 - Normal symmetrical movements \\
\hline 5a Motor Arm: Left Arm & $\begin{array}{c}1 \text { - Drift, limb holds } 90 \text { (or } 45 \text { ) degrees, but } \\
\text { drifts down before full } 10 \text { seconds, does not hit } \\
\text { the bed or other support }\end{array}$ \\
\hline 5b Motor Arm: Right Arm & $\begin{array}{l}0 \text { - No drift, limb holds } 90 \text { (or } 45 \text { ) degrees for } \\
\text { full } 10 \text { seconds }\end{array}$ \\
\hline 6a Motor Leg: Left Leg & 4 - No movement \\
\hline 6b Motor Leg: Right Leg & $\begin{array}{l}3 \text { - No effort against gravity, leg falls to bed } \\
\text { immediately }\end{array}$ \\
\hline 7 Limb Ataxia & 0 - Absent \\
\hline 8 Sensory & 0 - Normal, no sensory loss \\
\hline 9 Best Language & 0 - No aphasia. Normal \\
\hline 10 Dysarthria & $\begin{array}{c}0 \text { - Normal or intubated or another physical } \\
\text { barrier }\end{array}$ \\
\hline 11 Extinction and Inattention & $\begin{array}{c}1 \text { - Visual, tactile, auditory, spatial, or personal } \\
\text { inattention or extinction to simultaneous } \\
\text { bilateral stimulation in one of the sensory } \\
\text { modalities }\end{array}$ \\
\hline NIH Stroke Score Scale & 12 \\
\hline
\end{tabular}

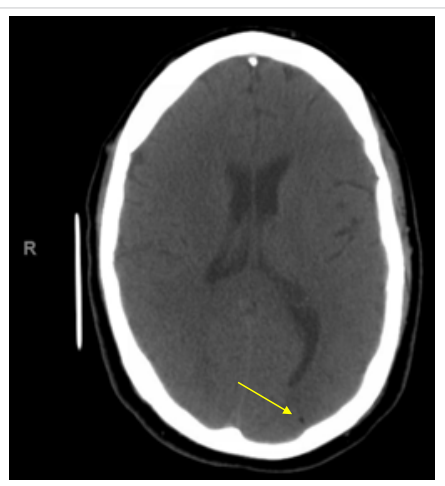

Figure 2: CT head without contrast 26 minutes after the end of the procedure showing the small hypodense area in the left occipital area that indicate an air embolus. 
the site of an epidural catheter unchanged from recent images done during the procedure, and no evidence of spinal cord compression was noted (Figure 3).

Repeated CT head and CT angiogram of head, neck and chest with contrast and 3-D reconstructions including images of the aortic arch and great vessels done 4 hours after the incident showed disappearance of the low density of gas bubble seen earlier in the left occipital cortex with development of a moderate size zone of low-attenuation consistent with acute infarction involving the left occipital lobe, inferior posterior left temporal lobe and right cerebellum (Figures 4,5). The vessels were patent, and no evidence of gas, dissection or thrombosis was seen.

Echocardiogram showed no evidence of air in the heart chambers with no intracardiac shunting, and the bubble study was negative. Hyperbaric oxygen chamber was not available at the hospital, and a decision was made not to transfer the patient to another hospital for HBOT due to the high risk involved in the transportation process, the amount of gas bubble found on the first CT head was very small and disappeared on subsequent CT head as well as the presence of pneumothorax and pneumomediastinum which are contraindications for HBOT unless the thoracostomy tube is placed first. MRI of the head and spine were unable to be done given recently inserted hook-wires in lungs. Follow up neurological exam demonstrates persistent right homonymous hemianopsia, paraplegia, lack of pain sensation

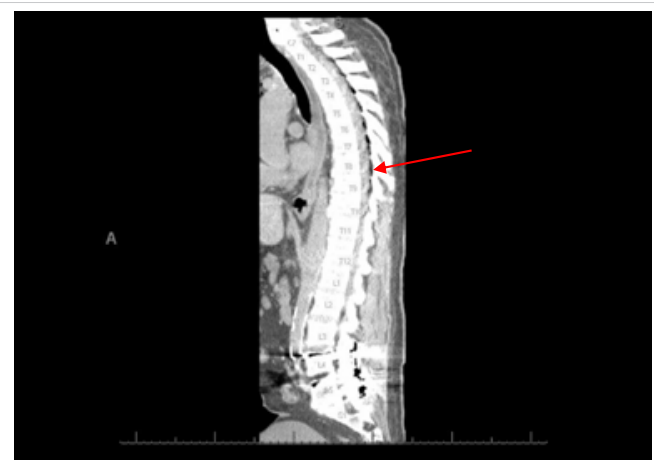

Figure 3: CT thoracic spine showed subcutaneous and epidural gas at the site of epidural catheter.

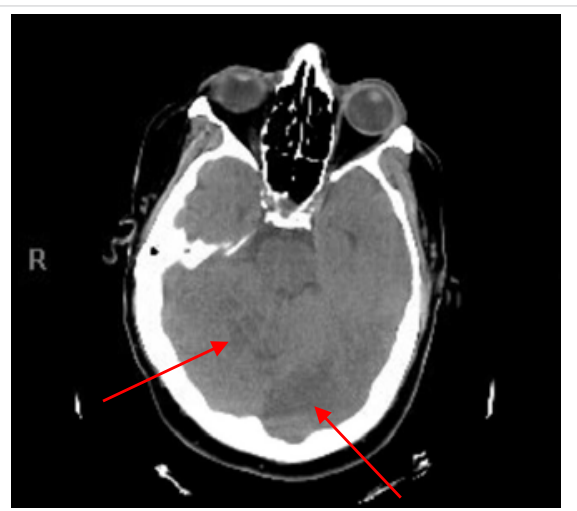

Figure 4: CT head with right superior cerebellar and left occipital lobe infarction.

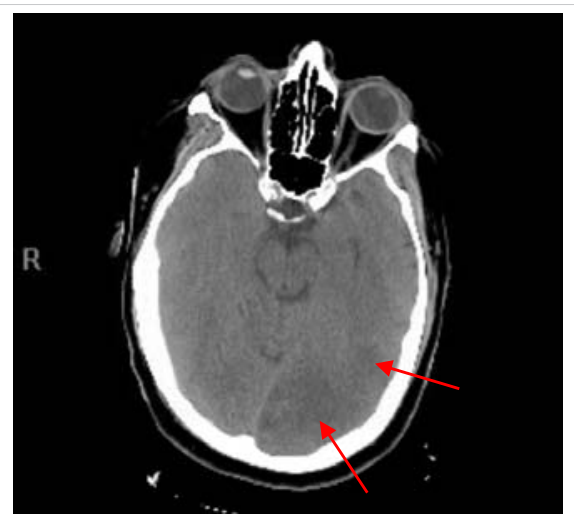

Figure 5: CT head with left occipital lobe and inferior posterior left temporal lobe infarction.

but preserved touch and proprioceptive sensation in the bilateral lower extremities along with a sensory level at T3, and +2 patella, +1 achilles deep tendon reflexes bilaterally. $\mathrm{He}$ was transferred to the Intensive care unit for monitoring. A few hours after arriving at the (ICU) the patient became restless, developed dysarthria, mild difficulty using his right hand, and started to exhibit right eye deviation with nystagmus. Continuous electroencephalogram with video showed severe encephalopathy and episodes of a clinical seizure lasting for 4 minutes originating from the left posterior head region accompanied by sharp delta epileptogenic activity with a background of diffuse slowing postictally.

The seizures were aborted with lorazepam and the patient was started on levetiracetam. After the seizures ceased, a new subtle right facial asymmetry was noted with persistent right homonymous hemianopsia and absence of voluntary movement in the bilateral lower extremities. Follow up serial CT of the head during hospital stay demonstrate moderate to large zone of acute and subacute infarctions involving the parasagittal left parietal and occipital lobes extending to near the left frontal lobe and the left parietal lobe laterally that marks the distribution of the posterior cerebral and anterior cerebral artery. Moderate zones of cerebellar infarction were also noted in the superior and inferior cerebellum and the involved area of the brain became slightly edematous with mild effacement of the fourth ventricle and left lateral ventricle but no midline shift or hemorrhage was noted (Figures 6-8).

Repeat CT of the Thoracic Spine showed resolution of previously noted epidural gas between T5-T9 with no obvious mass or hemorrhage. At this point, the patient had neurological presentation supporting the diagnosis of cerebral air embolism and anterior thoracic spinal cord syndrome due to systemic arterial micro air embolism in the anterior spinal artery.

Patient's neurological symptoms improved within the next few days with the exception of the loss of the paraplegia and absence of pain sensation in bilateral lower extremities, as well as development of bilateral ankle clonus. CT chest five days after the event showed dislodgment of localization wires. The 
patient remained neurologically and hemodynamically stable with mental status improvement. He underwent VATS with left upper lobe posterior segmentectomy with mediastinal and hilar lymph node dissection and removal of localization wires with intraoperative monitoring to avoid hypotension and hypoxia with uneventful recovery. Pathological examination of the removed segment of the lung showed large caliber blood vessels with surrounding emphysematous changes and malignant tumor cells consistent with fairly welldifferentiated adenocarcinoma of the lung with lipidic pattern (Figures 9,10).

MRI of the brain with Diffusion-weighted imaging (DWI) done two days after surgery showed multifocal areas of subacute infarction, the largest being in the posterior and medial aspect of the left occipital lobe. Multifocal punctate embolic type subacute areas of infarction were also noted in the right occipital lobe cortex, the splenium of the corpus callosum bilaterally, and in the frontal lobe cortex bilaterally but more so on the left without associated mass effect or hemorrhage (Figures 11-14).

Figures 11, 12, 13 and 14, MRI of the brain with Diffusion weight images showing multifocal punctate embolic type subacute areas of infarction involving multiple lobes of the brain bilaterally as well as large subacute lobar infarction in the posterior and medial aspect of the left occipital lobe.

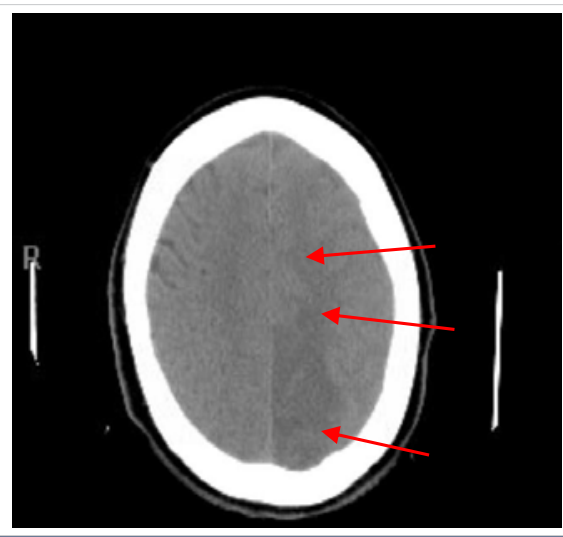

Figure 6: CT head showing low-attenuation areas of infarction involving parasagittal left parietal lobe extending to near the left frontal lobe.

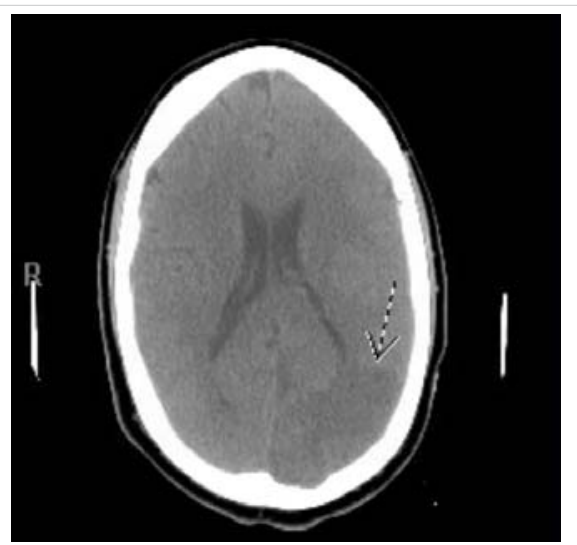

Figure 7: CT head showing low attenuation area of infarction involving left occipital lobe.

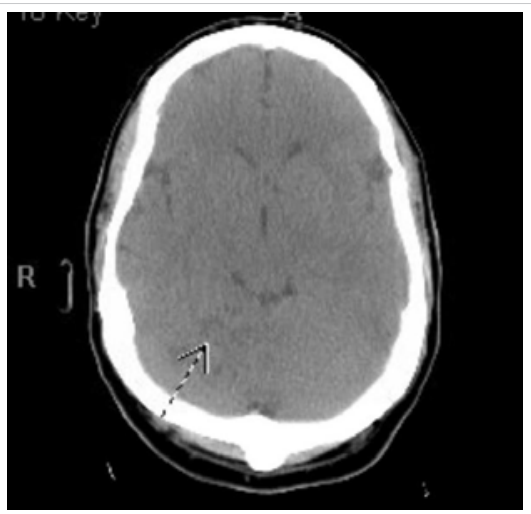

Figure 8: CT head showing low attenuation area of infarction in the right superior cerebellar.

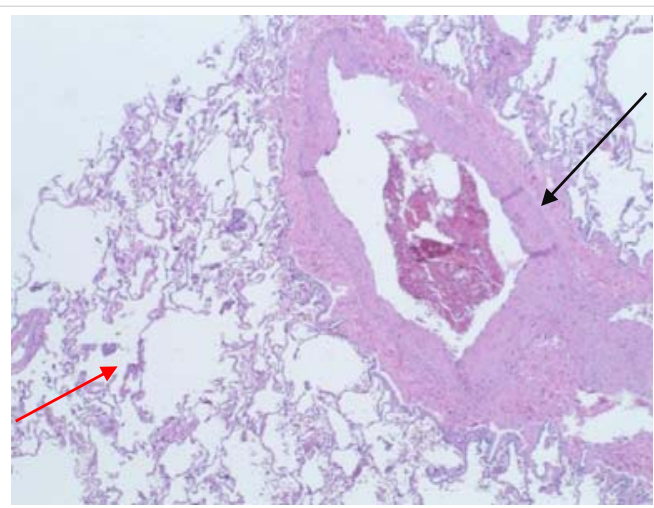

Figure 9: Large-caliber blood vessels (black arrow) with surrounding emphysematous changes (red arrow).

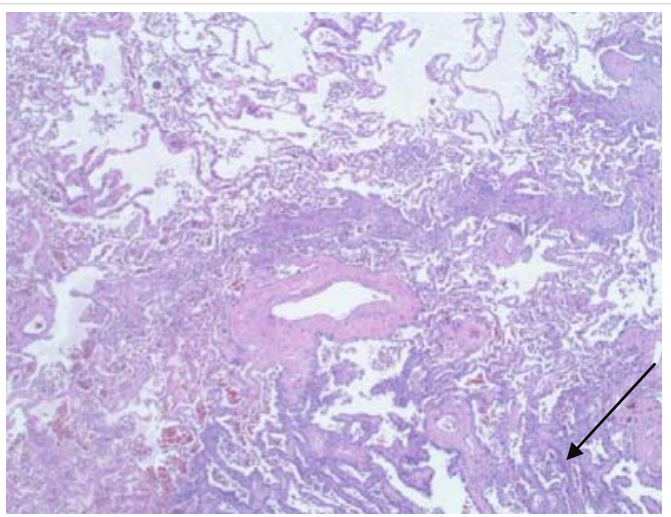

Figure 10: Malignant tumor cells consistent with fairly well-differentiated adenocarcinoma of the lung with lipidic pattern.

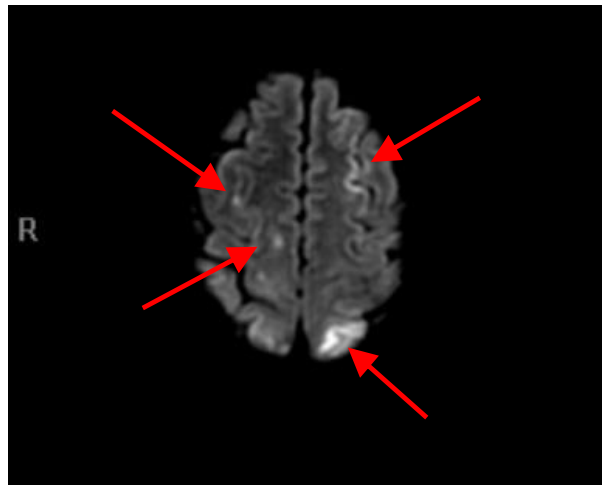

Figure 11: Brain MRI (DWI) 


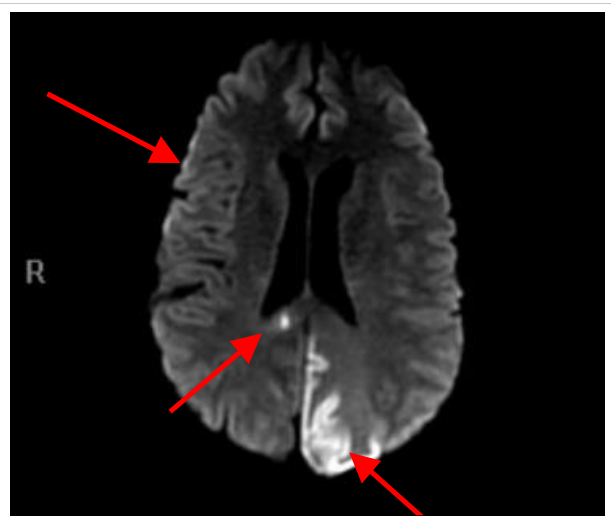

Figure 12: Brain MRI (DWI).

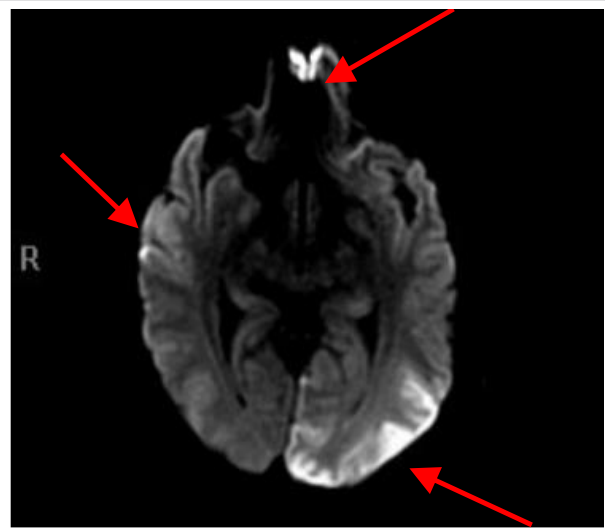

Figure 13: Brain MRI (DWI).

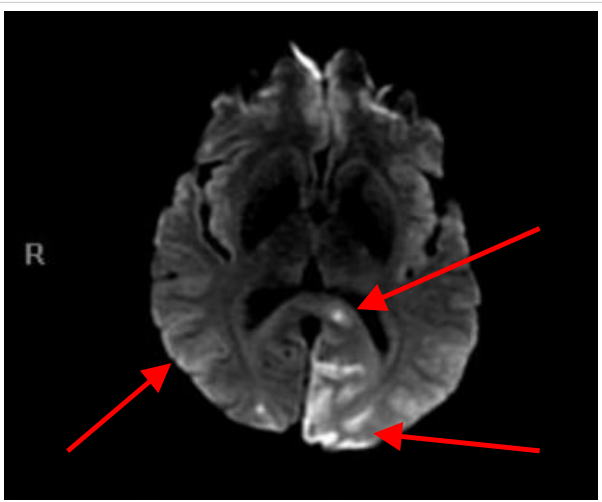

Figure 14: Brain MRI (DWI)

T2 Flair brain images on MRI showed subacute infarction involving left parietal, posterior and medial aspect of left occipital lobe (Figures 15,16).

MRI cervical, thoracic and lumbar spine with and without contrast with $\mathrm{T} 1, \mathrm{~T} 2$ sequence images and DWI showed no definite areas of cord signal abnormality and no cord compression or central canal stenosis (Figures 17,18).

Patient's neurological exam remained unchanged, and the patient was transferred to inpatient rehabilitation for physical therapy. Repeat MRI of thoracic spine three weeks later was still negative for ischemic changes of the spine. On discharge home he remained paraplegic with bladder and bowel incontinence requiring frequent bladder self-catheterization

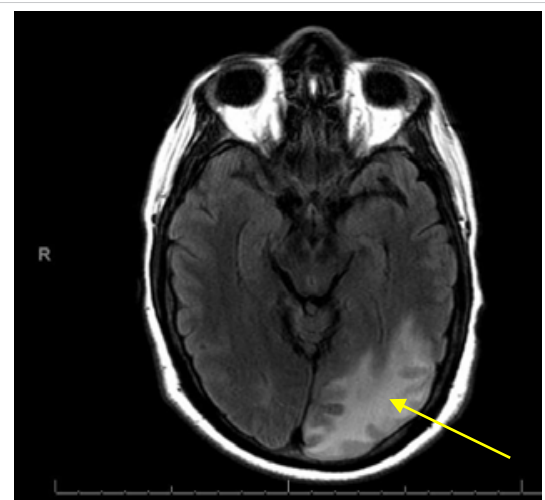

Figure 15: MRI Flair images of the brain showing subacute infarction involving left parietal occipital lobe.

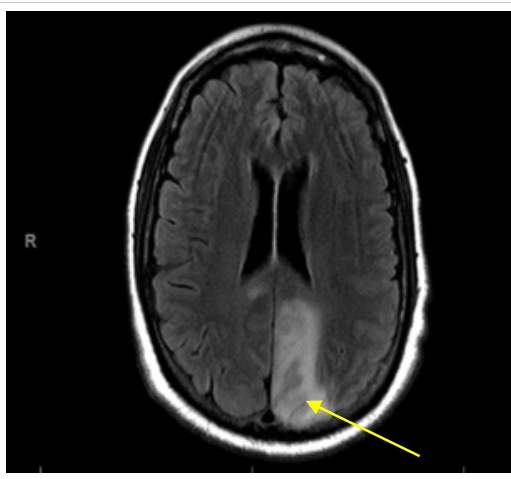

Figure 16: MRI Flair images of the brain showing subacute infarction involving medial aspect of left occipital lobe.

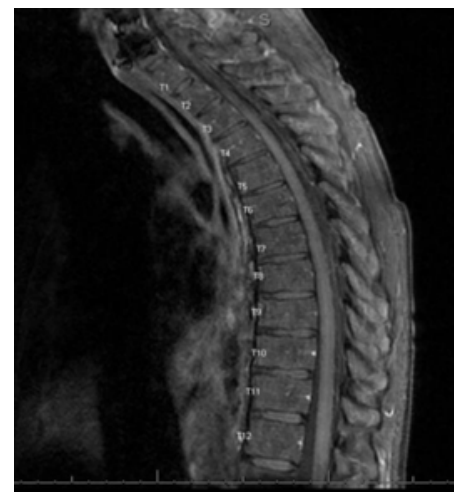

Figure 17: MRI thoracic spine with T1, T2 sequence Absence of stenosis or compression in the spinal cord.

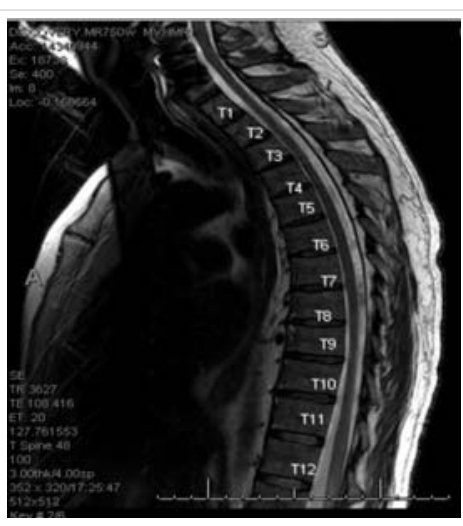

Figure 18: MRI Thoracic spine with T1, T2 sequence Absence of stenosis or compression in the spinal cord. 
but started to move his toes. The patient attended an outpatient rehabilitation program with notable improvement in his lower extremity strength and right visual field. At six months follow up his right lower extremity strength was $+3 / 5$ for hip flexion, $+2 / 5$ for knee extension and distal strength $+4 / 5$ while his left lower extremity strength was $+3 / 5$ for hip flexion, $+4 / 5$ for knee extension and distal strength $+5 / 5$. He was able to stand and walk with the assistance of a walker on his own for about 10 steps but continued to require regular intermittent bladder catheterization and remained on bowel regimen with regular bowel movements. The patient's clinical scenario remained most consistent with multiple micro air emboli to several sites, including cerebral arteries of the brain and anterior spinal artery.

\section{Discussion}

Computed tomography Hook wire localization of lung nodule is an invasive procedure performed by an interventional radiologist and often used preoperatively to VATS wedge resection to assist the surgeon in removing small and centrally located lung lesions [9]. The most common complications of this procedure are pneumothorax, intrapulmonary hemorrhage, hemoptysis and wire dislodgment, which are usually manageable with no serious sequel. However, systemic arterial air embolism (SAAE) is a very rare complication after hook wire localization with serious morbidity, poor patient outcome, and high mortality rate [10] with the underlying mechanism remaining largely unclear [11]. The incidence of SAAE after CT-guided lung biopsy is estimated to be $0.12 \%$ to $0.49 \%$ while after hook wire needle localization it is $0.02 \%-0.07 \%$. However, the real incidence of SAAE is probably higher since many asymptomatic cases are not diagnosed timely $[12,13]$. In many cases of SAAE, the air may remain in the heart and aorta without traveling distally to the vital organs. Under such circumstances, air get absorbed and resolve quickly without causing symptoms $[14,15]$.

The identification of intravascular air bubble by imaging study after the procedure can be missed as air may be rapidly absorbed from circulation. While resuscitating the patient is a priority and waiting for diagnostic images may take some time, the half-life of absorption of air is approximately 8 minutes, making SAAE diagnosis challenging. Once the air is in the systemic circulation, air embolism can lodge in distal vessels within any organ to cause ischemia. The most vulnerable of such organs are the brain, heart and spinal cord. The occlusion of functional end arteries by air embolus cause mechanical obstruction of the blood vessel leading to hypoxia and vasospasm which gets further aggravated by platelet activation and inflammatory response due to endothelial irritation resulting in impaired blood flow to the end organs [16]. SAAE after hook-wire localization occurs when air enters systemic circulation and for this to happen there are two requirements: (a) Direct communication between a source of air and the vasculature which can be a defect in the vessel wall created by the needle or wire, and (b) Pressure gradient which can be created by cough, deep inspiration, Valsalva maneuver, breath-hold or positive pressure ventilation which favors the passage of air through the defect into circulation rather than bleeding from the vessel.

The air that has entered the pulmonary vein goes to the left atrium first and then gets distributed distally to other organs through the systemic circulation. There are three suggested mechanisms by which air gains entry into the pulmonary vein during this procedure [17]. The first theory suggests that air may enter the pulmonary vein through alveolar- venous or bronchial-venous fistula created by the needle or the springing open hook wire as it injures through its pass the adjacent pulmonary vein and distal airway and can form a communication between them. When airway pressure exceeds pulmonary vein pressure such as with patient coughing, straining, or using Valsalva/holding the breath at end inspiration or if the patient has emphysema, this can facilitate air entry into pulmonary vein [18]. The second theory suggests air may enter the pulmonary vein directly via the puncture needle if the tip hits the pulmonary vein while the needle hub is not airtight and has direct exposure to atmosphere. This may occur, for instance, if not covered perfectly by operator's finger (as usually happens) or it is not filled with normal saline or gel while the wire is being introduced through the needle. When atmospheric pressure exceeds pulmonary vein pressure, such as when a patient takes a deep inspiration, then air will enter to the pulmonary vein. The third theory suggests air may enter first from the pulmonary artery if a needle tip hits the pulmonary artery branch and air enter from the atmosphere or distal airways when airway or atmospheric pressure exceeds pulmonary artery pressure, then reaches pulmonary vein circulation by traversing the pulmonary microvasculature.

Clinical presentation of systemic arterial air embolism (SAAE) depends on the distribution and location of the air emboli in the blood vessel and symptoms may be immediate or delayed with several hours after the procedure and some patients may even be asymptomatic [19]. Small amounts of gas embolism in the cerebrovascular or coronary artery can cause devastating sequela while other organs such as gastrointestinal and musculoskeletal can typically tolerate air embolism without significant symptoms. According to animal study, it is believed that only $2 \mathrm{ml}$ of air in the cerebral arteries and $0.5-1 \mathrm{ml}$ in the coronary arteries can be fatal. However, it is unknown how much air in the spinal artery can cause ischemia keeping in mind that the diameter of the anterior spinal artery ranges from $0.2-0.8$ $\mathrm{mm}$ so probably a very small amount of air in a spinal artery can cause ischemia to the spinal cord [20]. Patients with cerebral air embolism may present with rapid neurological deterioration including headache, loss of consciousness, dysarthria, confusion, seizure, motor deficit, visual changes, sensory deficit while cardiac presentation includes chest pain, 
bradycardia, arrhythmia, hypotension, myocardial infarction and cardiopulmonary arrest [21]. Spinal cord ischemia due to SAAE is extremely rare, and anterior spinal cord ischemia is more common than posterior ischemia. This is because only a single anterior spinal artery supplies the anterior two third of the spinal cord with fewer anastomoses, compared to the two posterior spinal arteries that have rich collateral circulation $[22,23]$. The neurological presentation of spinal cord ischemia due to SAAE depends on which spinal artery being affected. Anterior spinal cord syndrome (ACS) can present with sudden onset of bilateral loss of motor function of lower extremities associated with loss of pain and temperature perception but spares proprioceptive and vibratory sensation below the level of ischemia in the spinal cord. ACS can also lead to autonomic dysreflexia, which may entail bladder, bowel and sexual dysfunction and orthostatic hypotension [24].

Diagnosis of SAAE is mainly based on clinical findings and early recognition by operating physicians who should be aware of this possible rare complication during these invasive procedures [25]. MRI with diffusion-weighted imaging (DWI) is still the best imaging for detecting early brain ischemia; however, stroke with negative MRI findings is a well-recognized entity in particular in posterior circulation such as those seen in brainstem stroke [26,27]. Spinal MRI is considered the best imaging tool for the surrounding tissue around spinal cord but its sensitivity for detecting spinal cord ischemia is not $100 \%$ and MRI negative spinal cord ischemia has been reported [28]. One explanation for the negative ischemic finding of MRI is that the ischemic event of the spinal cord may have caused a slight reduction in blood flow that is severe enough to cause tissue oligemia and neurological symptoms but not severe enough to cause water shifting from extracellular to intracellular which is required for DWI MRI to detect as high signal indicating cell swelling and edema $[29,30]$. Serial MRI's are more helpful than a single MRI for detecting ischemic changes over time after initial ischemic event [31].

Risk factor for SAAE after CT guided hook wire localization and CT guided lung biopsy include lesion located above left atrium, deep lung lesion far from visceral pleura, patient movement during procedure or increase airway pressure which can occur during coughing, Valsalva maneuver or positive pressure ventilation, repositioning after procedure, deep inspiration [32]. Measures suggested to decrease risk of SAAE during these procedures include avoiding biopsy of cystic, cavitary or bullous lung lesion, not changing patient position suddenly after completing the procedure in addition to keeping needle hub occluded at all times, avoiding coughing and deep inspiration during procedure and penetrate the least amount of lung parenchyma by the needle [33]. Other conditions associated with intravascular air embolism are broad and include rapid ascent in scuba diving, barotrauma from positive pressure ventilation, head, neck, chest and abdominal blunt trauma, central and peripheral intravascular catheters/access, invasive procedures such Cardio-pulmonary bypass, Cardiac catheterization, ECHMO/Insertion of assist device, Cardiac ablation, pacemaker/defibrillator placement, OBGYN procedures such as cesarean section, hysteroscopy, and rarely after bronchoscopy and Endoscopic retrograde Cholangiopancreatography (ERCP).

A variety of surgeries can introduce air to venous and/ or arterial circulation if there is a patent foramen ovale (PFO). The major surgical interventions that can cause such complications include craniotomy, spinal surgery, carotid endarterectomy, Otolaryngological, cardiothoracic and abdominal surgeries. While transthoracic echocardiography (TTE) and transesophageal echocardiography (TEE) with bubble study (mixing saline with air and agitate the mixture to create microbubbles to inject in vein) are used mainly to detect the presence of (PFO) as an embolic source of stroke, it can be theoretically a potential source of cerebral air embolism in the presence of (PFO) or pulmonary shunt but with only few cases reported in the literature and unknown incidence rate of such a serious complication $[34,35]$.

Successful treatment of SAAE largely depends on early recognition and administration of supplemental oxygen with high fraction of inspired $\mathrm{FIO}_{2}$ at $100 \%$ by tight-fitting face mask or non-rebreather face mask which increases the partial pressure of oxygen in the blood leading to decreased partial pressure of nitrogen which leads to diffusion of nitrogen from inside the air bubble into the blood and tissue, thus reducing bubble size and increasing bubble absorption, in addition to supportive measures and positioning the patient safely in an attempt to prevent further air embolization to distal organs while optimal first-line treatment is immediate hyperbaric 0xygen therapy [36]. HBOT is the administration of $100 \%$ oxygen to a patient at a pressure greater than sea level usually at 2.5-2.8 atmosphere pressure in a chamber for one hour and up to 4 times a day [37]. It reduces air bubble volume by accelerating dissolution of nitrogen by replacing nitrogen with oxygen and increase oxygen solubility in the blood promoting the release of nitrogen in the air bubble into the blood and tissue and reduce vascular endothelial damage leading to more rapid restoration of blood flow within the blood vessel and provide oxygen to ischemic areas. It is the first-line therapy for treating a patient with arterial air embolism presenting with neurological deficit or end-organ damage, including patients with hemodynamic instability or compromised cardiovascular functions [38].

The presence of pneumothorax and pneumomediastinum is considered a contraindication to use HBOT unless the patient undergoes chest thoracotomy prior to application of HBOT. HBOT improves prognosis in patient with cerebral air embolism and reduces the mortality rate to 7\% [39]. Although immediate treatment with HBOT is recommended, delayed HBOT may also increase survival even 4 hours after the incidence of SAAE. However, the availability of HBOT is 
limited to certain regional hospitals and the potential risk of transporting a patient with SAAE to HBOT center, which may facilitate more air embolization to distal areas during transportation, should be weighed against the potential benefits. The side effects of HBOT may include pneumothorax, myopia, mild to severe pain from rupture of middle air tympanic membrane, the cranial sinuses and teeth, and generalized seizure. The optimal position for a patient with systemic arterial air embolism has remained controversial [40]. In venous air embolism, the left lateral decubitus or Trendelenburg position is proved to be effective by trapping air in the apex of the right ventricle and prevents its entry into the pulmonary artery system. There is little evidence of the ultimate position of a patient with arterial air embolism that could minimize the ischemic event and its associated complications [41].

Multiple positions are suggested for patient with arterial air embolism such as right lateral decubitus, Trendelenburg position, Trendelenburg with right lateral decubitus which may all be more effective if the air bubble is detected in the left ventricle and before it embolize distally by keeping the air away from the ventricular outflow tract [42]. The current recommendations are to avoid a sudden change in patient position and placing the patient in the supine position [43]. It has been reported that placing the patient with cerebral air embolism in Trendelenburg position has the potential to exacerbate cerebral edema $[44,45]$.

The mortality from SAAE was reported as high as $80 \%$ to $90 \%$ in the past, however, with more awareness of physicians and early diagnosis and application of HBOT the mortality rate recently decreased to $21 \%$ [46]. Gradual neurological improvement after cerebral ischemic stroke and spinal cord ischemia due to arterial air embolism is not uncommon with the aggressive rehabilitation program, and it may continue long after hospital discharge [47]. Studies suggest that $40 \%$ of patients who survive SAAE are wheelchair-bound upon discharge. However, $40 \%$ of these patients will be able to walk independently in the future, but some of them would not recover due to the underlying neurological deficits [48]. Full recovery is possible in minority of patients and sparing of proprioceptive sensation is considered a good prognostic factor for patients presenting with ACS [49]. It is reasonable to assume that our patient suffered from cerebral and anterior thoracic spinal cord ischemia which had resulted from multiple arterial air embolisms during the hook wire localization of his lung lesions.

\section{Conclusion}

Systemic arterial air embolism (SAAE) is rare but can be a fatal complication during or after invasive procedures such as CT guided lung biopsy and hook wire localization and may lead to devastating outcome [50]. It is important for the operator of these invasive procedures, usually an interventional radiologist, as well as physicians responding to codes and stroke alert teams to maintain a high level of suspicion for SAAE and recognize its clinical presentation and be familiar with its unique but limited management approach which includes maintaining safe positioning for patient and application of $100 \%$ oxygen supplement while arranging for immediate HBOT when available or identifying the nearest facility with HBO chamber to transfer patient when he is stable [51]. Early application of HBOT plays an important role in the clinical outcome [52]. Ideally, HBOT should be available on-site at the same facility where these invasive procedures with high-risk for SAAE are performed to avoid any delay during interhospital patient transfers [53]. Absence of air on computed tomography imaging after the occurrence of SAAE should not delay the decision to treat these patients with HBOT as air can be rapidly absorbed from circulation [54]. Preventive measures to avoid or minimize this complication during these procedures remain the optimum goal [55].

\section{Acknowledgment}

The authors thank Atif Hashmi MD for reviewing the manuscript and for his valuable constructive suggestions and support.

\section{References}

1. Shin KM, Lim JK, Kim CH. Delayed presentation of cerebellar and spinal cord infarction as a complication of computed tomographyguided transthoracic lung biopsy: a case report. J Med Case Rep. 2014; 8: 272.

PubMed: https://pubmed.ncbi.nlm.nih.gov/25113016/

2. Jang $H$, Rho JY, Suh YJ, Jeong YJ. Asymptomatic systemic air embolism after CT-guided percutaneous transthoracic needle biopsy. Elsevier Clin Imaging. 2019; 53: 49-57.

PubMed: https://pubmed.ncbi.nlm.nih.gov/30312855/

3. Kume A, Yoneyama S, Takahashi A, Watanabe, H. MRI of anterior spinal artery syndrome. J Neurol Neurosurg Psychiatry. 1992; 55: 838-840. PubMed: https://pubmed.ncbi.nlm.nih.gov/1402978/

4. Boddu SR, Cianfoni A, Kim KW, Banihashemi MA, Pravata Em, et al. Spinal Cord Infarction and Differential Diagnosis. Neurovascular Imaging.

5. Blanc $P$, Boussuges A, Henriette K, Sainty JM, Deleflie M. latrogenic cerebral air embolism: the importance of early hyperbaric oxygenation. Intensive Care Med. 2002; 28:559-563.

PubMed: https://pubmed.ncbi.nlm.nih.gov/12029402/

6. Yi JH, Choi PJ, Bang JH, Jeong SS, Cho JH. Systemic air embolism after computed tomography-guided hook wire localization: two case reports and literature review. J Thorac Dis. 2018; 10: E59-E64. PubMed: https://pubmed.ncbi.nlm.nih.gov/29600106/

7. O’Dowd, LC, Kelley, MA. Air embolism. UpToDate. 2019; 1-39

8. Thomas R, Thangakunam B, Cherian RA, Gupta R, Christopher DJ. Cerebral air embolism complicating CT-guided transthoracic needle biopsy of the lung. Clin Respirat J. 2011; e1-e3. PubMed: https://pubmed.ncbi.nlm.nih.gov/21410896/

9. Gossot D, Miaux Y, Guermazi A, Celerier M, Friga J. The hook-wire technique for localization of pulmonary nodules during thoracoscopic resection. Chest. 1994; 105: 1467-1469.

PubMed: https://pubmed.ncbi.nlm.nih.gov/8181339/

10. Horan T, Pinheiro P, Araujo LM, Santiago, FF, Rodrigues MR. Massive Gas Embolism During Pulmonary Nodule Hook Wire Localization. Ann Thorac Surg. 2002; 73: 1647-1649.

PubMed: https://pubmed.ncbi.nlm.nih.gov/12022575/ 
11. Sakiyama S, Kondo K, Matsuoka H, Yoshida M, Miyoshi T, et al. Fatal air embolism during computed tomography-guided pulmonary marking with a hook-type marker. J Thorac Cardiovasc Surg. 2003; 126: 1207-1209. PubMed: https://pubmed.ncbi.nlm.nih.gov/14566279/

12. Sun $D$, Sui $P$, Zhang $W$, Zhang L, Xu H. Cerebral air embolism during percutaneous computed tomography scan-guided liver biopsy. J Can Res Ther. 2018; 14: 1650-1654.

PubMed: https://pubmed.ncbi.nlm.nih.gov/30589054/

13. Sato K, Miyauchi K, Shikata F, Murakami T, Yoshioka S, Kawachi K. Arterial Air Embolism during Percutaneous Pulmonary Marking under Computed Tomography Guidance. Jpn J Thorac Cardiovasc Surg. 2005; 53: 404-406.

PubMed: https://www.ncbi.nlm.nih.gov/pubmed/16095246

14. Tagawa T, Takasu M, Ishida T. Asymptomatic Air Embolism After Computed Tomography Guided Lung Needle Marking. J Thorac Oncol. 2009; 4: 937-938.

PubMed: https://www.ncbi.nlm.nih.gov/pubmed/19550251

15. Tagawa T, Takasu M, Ishida T. Asymptomatic Air Embolism After Computed Tomography-Guided Lung Needle Marking. J Thorac Oncol. 2009; 4: 938-938.

PubMed: https://www.ncbi.nlm.nih.gov/pubmed/19550251

16. MuthCM,ShankES. GasEmbolism. NewEnglJMed. 2000;342:476-482. PubMed: https://pubmed.ncbi.nlm.nih.gov/10675429/

17. Wang MY, Liu YS, An XB, Li K, Liu YJ, et al. Cerebral arterial air embolism after computed tomography-guided hook-wire localization of a pulmonary nodule. Med. 2019; 98: e15437.

PubMed: https://pubmed.ncbi.nlm.nih.gov/31045810/

18. Arnold BW, Zwiebel WJ. Percutaneous Transthoracic Needle Biopsy Complicated by Air Embolism. AJR. 2002; 178: 1400-1402

PubMed: https://pubmed.ncbi.nlm.nih.gov/12034605/

19. Shi L, Zhang R, Wang Z, Zhou P. Delayed Cerebral Air Embolism Complicating Percutaneous Needle Biopsy of the Lung. Am J Med Sci. 2013; 345: 501-503.

PubMed: https://pubmed.ncbi.nlm.nih.gov/23276901/

20. Kaichi Y, Kakeda S, Korogi Y, Nezu T, Aoki S, et al. Changes over Time in Intracranial Air in Patients with Cerebral Air Embolism: Radiological Study in Two Cases. Case Rep Neurol Med. 2015; 491017 PubMed: https://pubmed.ncbi.nlm.nih.gov/26640730/

21. Fiore L, Frenk NE, Martins GLP, Viana PCC, de Menezes MR. Systemic Air Embolism after Percutaneous Lung Biopsy: A Manageable Complication. Radiol Case. 2017; 11: 6-14.

PubMed: https://pubmed.ncbi.nlm.nih.gov/29299093/

22. Djurberg $\mathrm{H}$, Haddad M. Anterior spinal artery syndrome; Paraplegia following a segmental ischaemic injury to the spinal cord after oesophagectomy. Anaesthesia. 1995; 50: 345-348.

PubMed: https://pubmed.ncbi.nlm.nih.gov/7747855/

23. Klakeel M, Thompson J, Srinivasan R, McDonald F. Anterior spinal cord syndrome of unknown aetiology. Proc (Bayl Univ Med Cent) 2015 28: 85-87.

PubMed: https://pubmed.ncbi.nlm.nih.gov/25552812/

24. Vargas MI, Gariani J, Sztajzel R, Barnaure-Nachbar I, Delattre BM, et al. Spinal Cord Ischemia: Practical Imaging Tips, Pearls, and Pitfalls. Am J Neuroradiol. 2015; 36: 825-830.

PubMed: https://pubmed.ncbi.nlm.nih.gov/25324492/

25. Wu CC, Maher MM, Shepard JA. Complications of CT-Guided percutaneous Needle Biopsy of the Chest: Prevention and management. AJR. 2011; 196: W678-W682.

PubMed: https://pubmed.ncbi.nlm.nih.gov/21606253/

26. Watts J, Wood B, Kelly A, Alvaro A. Stroke syndromes associated with $\mathrm{DWI}$-negative $\mathrm{MRI}$ include ataxic hemiparesis and isolated internuclear ophthalmoplegia. Am Acad Neurol. 2013; 186-191.

PubMed: https://pubmed.ncbi.nlm.nih.gov/29473634/
27. Edlow BL, Hurwitz S, Edlow JA. Diagnosis of DWI-negative acute ischemic stroke A meta-analysis. Neurol. 2017; 89: 256-262.

PubMed: https://pubmed.ncbi.nlm.nih.gov/28615423/

28. Thurnher MM, Bammer R. Diffusion-weighted MR imaging (DWI) in spinal cord ischemia. Neuroradiology. 48: 795-801. PubMed: https://pubmed.ncbi.nlm.nih.gov/16977443/

29. Makin SDJ, Doubal FN, Dennis MS, Wardlaw JM. Clinically Confirmed Stroke With Negative Diffusion-Weighted Imaging Magnetic Resonance Imaging. Stroke. 2015; 46: 3142-3148.

PubMed: https://pubmed.ncbi.nlm.nih.gov/26419965/

30. Kume A, Yoneyama S, Takahashi A, Watanabe H. MRI of anterior spinal artery syndrome. J Neurol Neurosurg Psychiatry. 1992; 55: 838-840. PubMed: https://pubmed.ncbi.nlm.nih.gov/1402978/

31. Reale G. Not all that glitters is gold: MR-DWI negative ischemic stroke. 2017. https://eso-stroke.org/strokeresearch/mr-dwi-negative-ischemicstroke-not-all-strokes-glitter-in-dwi-sequences/.

32. Rocha RD, Azevedo AA, Falsarella PM, Rahal Jr A, Garcia RG. Cerebral air embolism during CT-guided lung biopsy. Thorax. 2015; 70: 1099-1100.

PubMed: https://pubmed.ncbi.nlm.nih.gov/26024689/

33. Shin KM, Lim JK, Kim CH. Delayed presentation of cerebellar and spinal cord infarction a complication of computed tomography-guided transthoracic lung biopsy: a case report. J Med Case Rep. 2014; 8: 272. http://www.jmedicalcasereports.com/content/8/1/272.

34. Romero JR, Frey JL, Schwamm LH, Demaerschalk BM, Chaliki $\mathrm{HP}$, et al. Cerebral Ischemic Events Associated with "Bubble Study" for Identification of Right to left shunts. Strok. 2009; 40: 2343-2348. PubMed: https://pubmed.ncbi.nlm.nih.gov/19498192/

35. Dedaj R, Preston IR. A case report: acute Stroke After Echocardiogram with bubble study in A patient with Patent Foramen Ovale and Chronic Thromboembolic Pulmonary hypertension. Am J Respir Crit Care Med. 2012; 185: A6170.

36. McCarthy CJ, Behravesh S, Naidu SG, Oklu R. Air Embolism: practical tips for prevention and treatment. J Clin Med. 2016; 93: 1-13. PubMed: https://pubmed.ncbi.nlm.nih.gov/27809224/

37. Balsara ZN, Burks DD. Hyperbaric Oxygen Therapy for Arterial Air Embolism. AJR. 2007; 188: W98-W98.

PubMed: https://pubmed.ncbi.nlm.nih.gov/17179337/

38. Leach RM, Rees PJ, Wilmshurst P. Hyperbaric Oxygen therapy. BMJ. 1998; 317: 1140.

PubMed: https://pubmed.ncbi.nlm.nih.gov/9784458/

39. Bou-Assaly W, Pernicano P, Hoeffner E. Systemic air embolism after transthoracic lung biopsy: A case report and review of the literature. World J Radiol. 2010; 28: 193-196.

PubMed: https://www.ncbi.nlm.nih.gov/pmc/articles/PMC2999016/

40. Al-Ali WM, Browne T, Jones R. A Case of Cranial Air Embolism after Transthoracic Lung Biopsy. Am J Respirat Critical Care Med. 2012; 186: 1193-1195.

41. Hiraki T, Fujiwara H, Sakurai J, Iguchi $T$, Gobara H, et al. Nonfatal Systemic Air Embolism Complicating Percutaneous CT-Guided Transthoracic Needle Biopsy: Four Cases From a Single Institution. Chest. 2007; 132: 684-690.

PubMed: https://pubmed.ncbi.nlm.nih.gov/17699141/

42. Bou-Assaly W, Pernicano P, Hoeffner E. Systemic air embolism after transthoracic lung biopsy: A case report and review of the literature. WJR. 2010; 2: 193-196.

PubMed: https://www.ncbi.nlm.nih.gov/pmc/articles/PMC2999016/

43. Mansour A, Abdel Raouf S, Qandeel M, Swaidan M. Acute Coronary Artery Air Embolism Following CT-Guided Lung Biopsy. Cardiovasc Intervent Radiol. 2005; 28: 131-134.

PubMed: https://pubmed.ncbi.nlm.nih.gov/15602640/ 
44. McCarthy CJ, Behravesh S, Naidu SG, Oklu R. Air Embolism: Practical Tips for Prevention and Treatment. J Clini Med. 2016; 5: 93. PubMed: https://pubmed.ncbi.nIm.nih.gov/27809224/

45. Moon RE, de Lisle dear G, Stolp BW. Treatment of decompression illness and iatrogenic gas Embolism. Respir Care Clin N Am. 1999; 5: 93-135. PubMed: https://pubmed.ncbi.nlm.nih.gov/10205814/

46. Chuang DY, Sundararajan S, Sundararajan VA, Feldman DI, Xiong W. Accidental Air Embolism. An Uncommon cause of latrogenic Stroke. Stroke. 2019; 50: e183-e186.

47. Robertson CE, Brown Jr RD, Wijdicks EFM, Rabinstein AA. Recovery after spinal cord infarcts/Long-term outcome in 115 patients. Neurology. 2012; 78: 114-121.

PubMed: https://pubmed.ncbi.nlm.nih.gov/22205760/

48. Masson C, Pruvo JP, Meder JF, Cordonnier C, Touze E, et al. Spinal cord infarction: clinical and magnetic resonance imaging findings and shortterm outcome. J Neurol Neurosurg Psychiatry. 2004; 75: 1431-1435. PubMed: https://www.ncbi.nlm.nih.gov/pmc/articles/PMC1738740/

49. Nedeltchev K, Loher TJ, Stepper F, Arnold M, Schroth G, et al. LongTerm Outcome of Acute Spinal Cord Ischemia Syndrome. Stroke. 2004; 35: 560-565.

PubMed: https://pubmed.ncbi.nlm.nih.gov/14726546/

50. Sakiyama S, Kondo K, Matsuoka H, Yoshida M, Miyoshi T, et al. Fatal Air embolism during computed tomography-guided pulmonary marking with a hook-type marker. J Thorac Cardiovasc Surg. 2003; 126: 1207-1209. PubMed: https://pubmed.ncbi.nlm.nih.gov/14566279/

51. Hellinger L, Keppler AM, Schoeppenthau H, Perras J, Bender R. Hyperbaric oxygen therapy for iatrogenic arterial gas embolism after CT-guided lung biopsy. Anaesthesist. 2019; 68: 456-460. PubMed: https://pubmed.ncbi.nlm.nih.gov/31264050/

52. Arai H, Tajiri M, Ando K, Okudela K, Iwasawa T, Masuda M. Possible Complication of Marking Hook Wire Entry into the Systemic Circulation during Video-Assisted Thoracoscopic Surgery. Int J Respir Pulm Med. 2016; 3: 060.

53. Lattin G, O'Brien W, McCrary B, Kearney P, Gover D, et al. Massive systemic Air Embolism treated with Hyperbaric Oxygen Therapy Following CT-guided Transthoracic Needle Biopsy of a Pulmonary Nodule. J Vasc Interv Radiol. 2006; 17: 1355-1358. PubMed: https://pubmed.ncbi.nlm.nih.gov/16923984/

54. Brito C, Graca J, Vilela P. Cerebral Air Embolism: The importance of Computed Tomography Evaluation. J Med Cases. 2020; 11: 394-399.

55. Goldney B, Schonherr E, Freund MC, Haslauer M, Petersen J, et al. Measures to prevent Air Embolism in transthoracic Biopsy of the lung. AJR Am J Roentgenol. 2017; 208: W184-W191.

PubMed: https://pubmed.ncbi.nlm.nih.gov/28301208/ 